\title{
MOLECULES WITH HIGH ROTATIONAL ANGULAR MOMENTUM: A GENERALIZED AB INITIO APPROACH*
}

\author{
LAWRENCE L. LOHR \\ Department of Chemistry, University of Michigan, Ann Arbor, MI 48109 (U.S.A.)
}

(Received 17 January 1989)

\begin{abstract}
An outline is given of a systematic and automated computational procedure based upon our successful ab initio approach to the theoretical study of centrifugal distortions of rotating molecules and their associated energy stabilizations. Such a generalized procedure permits the ready evaluation of the centrifugal distortion spectroscopic constants for moderate-sized molecules of low or no symmetry; these molecules represent cases for which the older version of our computational procedure is unwieldy. More broadly, we explore the energies and geometries of molecules in those regions of nuclear coordinate hyperspace which are accessible by such distortions from the equilibrium geometry. A method is outlined for optimizing the geometry of a molecule in the presence of a centrifugal field which is parametrically dependent upon the rotational angular momentum. The field is not fixed for a given angular momentum, but is coupled with the geometry via the moment of inertia tensor. This development should prove to be of major benefit to both computational chemists and molecular spectroscopists, as it will advance the interplay of theoretical and experimental studies of molecular structure.
\end{abstract}

\section{INTRODUCTION}

The distortion of a rotating molecule from its equilibrium geometry and the effect of the distortion upon the energy levels has been recognized for a long time by molecular spectroscopists. Interest in these centrifugal effects, as they are often called, has increased in recent years due to the development of highresolution spectroscopic techniques and to major advances in the theoretical description of highly excited rotational states of molecules. A particular focus of interest [1-5] has been the spherical top $\mathrm{SF}_{6}$, as this molecule displays splittings of the $(2 J+1)^{2}$ degenerate rigid rotor levels into clustered patterns. The concept [5] of a rotational energy surface portraying the variation in the rotational energy with respect to the direction of the angular momentum in a body-fixed frame has proven to be particularly useful in understanding these patterns.

*Dedicated to Professor Per-Olov Löwdin. 
In a series of recent studies [6-9] we presented a new approach to centrifugal distortions and their associated rotational energy stabilizations which exploits ab initio electronic structure computational methods. The approach is direct, bypassing in the simplest applications the explicit calculation of spectroscopic constants such as vibrational frequencies as this information is implicitly contained in the ab initio electronic energy hypersurface. Specifically, the method is particularly useful at any computational level for which analytic gradients of potential-energy hypersurfaces are available. Results have been presented previously [6] for such calculations on $\mathrm{H}_{2}^{+}, \mathrm{NH}_{3}, \mathrm{CH}_{4}, \mathrm{BF}_{3}$ and $\mathrm{SF}_{6}$. More detailed studies [7-9] of $\mathrm{H}_{2} \mathrm{O}, \mathrm{O}_{3}$, and $\mathrm{PH}_{3}$ have also been reported. The procedure is structurally oriented, i.e. it focusses on the question of the size and shape of molecules with non-zero rotational angular momentum. Centrifugal distortion spectroscopic constants are a very useful form of the computational output, providing an important and indispensable basis for comparison to experimental observations, yet their computation is, in a way, secondary to the main task. In other words, our studies are an exploration of molecular energy in those regions of the nuclear coordinate hyperspace which are accessible by centrifugal distortions from the equilibrium geometry.

\section{BASIC METHOD}

We first review the basic form of the approach to rotationally induced distortions which we have used in our earlier studies, and we then outline the extensions and generalizations which comprise the basis of the new method. The key feature in both the earlier and the present versions of the method is the exploitation of the current availability at many levels of quantum chemical computation of analytic gradients of the total electronic energy with respect to nuclear coordinates. Taking the molecule to be a classically rotating deformable body with quantum mechanical internal forces, the basic problem is to locate quasi-equilibrium structures, i.e. structures which satisfy the requirements that (i) the gradient of the sum of the electronic and rotational energies is zero and (ii) the Hessian (second-derivative) matrix of this effective potential energy has all positive eigenvalues. The resulting structure and its energy are parametrically dependent on both the magnitude and the direction of the rotational momentum in the molecular frame. It should be noted that there is no need to reduce the results to a conventional power series representation of the dependence of the energy upon the rotational quantum numbers. In fact, it is more accurate, particularly for a very high angular momentum value, to present the dependence directly by means of tables or graphs. In other words, within the assumptions of classical rotation and of a selected quantum-chemical level of electronic description, the dependence of the energy upon the rotational angular momentum is obtained exactly.

In our first studies [6], we considered only spherical and symmetric top 
molecules; with rotations taken about the principal axes the spherical tops in effect become symmetric tops, while the symmetric tops remain as such for rotations about the principal axis coincident with the top axis. In many of the examples, the rotational energy depended via the moment of inertia upon only one suitably chosen internal coordinate. For example, with $\mathrm{J}$ parallel to $\mathrm{C}_{4}$ for $\mathrm{SF}_{6}$, such that the effective symmetry is $D_{4 \mathrm{~h}}$, there are two independent structural variables, namely the equatorial and axial bond lengths, but the rotational energy depends only on the former. Thus the computational task is particularly simple: select a value of the equatorial bond length in excess of its equilibrium value, carry out a constrained geometrical optimization with respect to the remaining variable or variables (only the axial bond length in this case), calculate the (non-vanishing) gradient with respect to the fixed equatorial bond length, calculate the magnitude of rotational angular momentum about $\mathrm{C}_{4}$ needed to produce a rotational-energy gradient equal in magnitude but opposite in sign, and finally calculate the total (electronic plus rotational) energy for this $\mathbf{J}$. A range of displacements produces a range of associated $\mathbf{J}$ values, thus defining centrifugal distortion pathways. For $\mathrm{SF}_{6}$ the calculations were also done with $\mathrm{J}$ parallel to $\mathrm{C}_{3}$, thus enabling the calculation of both scalar $\left(D_{\mathrm{s}}\right)$ and tensor $\left(D_{\mathrm{t}}\right)$ quartic centrifugal distortion coefficients. Values of these coefficients have been obtained [6] at the HF/6-31G* level: $D_{\mathrm{t}}=5.68 \times 10^{-9}$ and $D_{s}=-2.0 \times 10^{-10} \mathrm{~cm}^{-1}$ as compared with experimental [3] values of $D_{\mathrm{t}}=(5.54743 \pm 0.00043) \times 10^{-9}$ and $D_{\mathrm{s}}=-(1.8994 \pm 0.0064) \times 10^{-10} \mathrm{~cm}^{-1}$. One of the successes of the method [6] is that we were able to reject other experimental values of $D_{\mathrm{s}}$, ranging from a factor of three greater to a factor of ten smaller, as being unreasonable and, therefore, to determine the most likely artifacts of spectral fitting procedures. Most of our earlier calculations were made at the self-consistent field (HF) level which turns out to be reasonably satisfactory for two reasons: (i) that bond distances are underestimated (particularly so with the $6-31 \mathrm{G}^{* *}$ basis set) and thereby cause the rotational constants to be overestimated; and (ii) that restoring forces are overestimated. Thus quartic centrifugal distortion constants, which vary as the cube of a rotational constant divided by the square of a vibrational frequency (or by the first power of a spring constant), have roughly cancelling errors.

In cases such as the rotation of the symmetric top species $\mathrm{NH}_{3}$ and $\mathrm{PH}_{3}$ about a principal axis perpendicular to the top axis, the moment of inertia, and hence the rotational energy, depends upon more than one structural variable. This situation necessitates the simultaneous solution of two, or in general more, equations that represent the vanishing of the gradient of the sum of electronic and rotational energies; i.e. distortions must be sought such that they lie on the centrifugal pathway. Although we have done this not only for these two molecules but also for the asymmetric tops $\mathrm{H}_{2} \mathrm{O}$ and $\mathrm{O}_{3}$, for which a similar situation occurs when the angular momentum is perpendicular to the molec- 
ular plane, the procedure is awkward and not easily implemented for larger asymmetric tops.

Accordingly, it is a key part of the generalized method to have a more feasible computational procedure for studying rotationally induced distortions and for calculating spectroscopic distortion coefficients for larger molecules not necessarily possessing elements of symmetry. It should be noted that our procedures are applicable only to rotations about principal axes, as then the angularmomentum and angular-velocity vectors are parallel and thus correspond to a dynamic balance. The instability associated with rotation about the intermediate axis of an asymmetric top is not a difficulty, as the effective energy is nonetheless stationary; i.e. $J$ parallel to the intermediate axis corresponds to a stationary point on the rotational-energy surface.

The generalized procedure differs from the one previously used in that it is inverted; i.e. the classical angular momentum components $J_{x}, J_{y}$ and $J_{z}$ are specified, the rotational energy and its gradient are calculated for the trial molecular geometry, the electronic energy and its gradient are calculated at the selected quantum-chemical level at the same geometry, and a stationary point on the effective surface is then sought according to a standard algorithm. The resulting structure and total (electronic plus rotational) energy thus depend parametrically on the rotational-angular-momentum vector. At first it may appear that this procedure is suspect in that it treats rotation explicitly while ignoring vibration, thus putting the small before the large. However, this order is identical to that used in the standard treatment of electronic wave functions for the hydrogen atom, where the radial ("vibrational") energies and wave functions are solutions of different effective Schrödinger equations for different angular momenta. Nonetheless, it is very important, particularly for hydride molecules, to consider an averaging over the vibrational motion even for vibrational ground states. The entire procedure can be formulated directly or indirectly in terms of the commonly used internal $\mathbf{Z}$ matrix coordinates, thus facilitating the application of the available computer codes for electronic energy gradients.

As is well known for diatomic molecules, there are both minimal and maximal stationary points in effective potential-energy curves for $J$ values below some critical value. Above this value there are no stationary points (excepting the limit of infinite internuclear separation). In our studies to date the quasiequilibrium structures were typically close to the "true" equilibrium geometries (for $J=0$ ) and the molecules themselves were all fairly rigid (large vibrational frequencies), so that stationary points were assumed to be minima. However, it is important, particularly for "floppy" (non-rigid) molecules, to compute the Hessian matrix, $\mathbf{H}$, and its eigenvalues at the stationary points of the effective potential-energy surfaces. Indeed the method enables the exploration of the geometries and energies of stationary points other than minima on effective potential-energy hypersurfaces for polyatomic molecules. 
We now outline the method of incorporating rotational energies into geometry optimization algorithms. The rotational energy depends through the moment of inertial tensor upon the set of nuclear coordinates. Therefore it may be thought of as an augmentation of the nuclear repulsion energy; for a specified direction and magnitude of $\mathbf{J}$ there is added to the energy

$E_{\mathbf{r}}(\mathbf{Q}, \mathbf{J})=\mathrm{A}(\mathbf{Q}) J_{x}^{2}+B(\mathbf{Q}) J_{y}^{2}+C(\mathbf{Q}) J_{z}^{2}$

where $A, B$ and $C$ are the $\mathbf{Q}$ dependent rotational constants, with $\mathbf{Q}$ being the set of nuclear coordinates. The directions $x, y$ and $z$ are taken as the principal axes of the moment-of-inertia tensor $\mathbf{I}(\mathbf{Q})$. More generally, the rotational energy may be represented as

$E_{\mathrm{e}}(\mathbf{Q}, \mathbf{J})=1 / 2 \mathbf{J I}-1 \mathbf{J}$

where $\mathbf{I}^{-1}$ is the inverse moment-of-inertia tensor. Many geometry optimization (total energy minimization) schemes require analytic gradients; the gradient of $E_{\mathrm{r}}$ is calculated and added to the gradient of the Born-Oppenheimer electronic energy $E_{\mathrm{e}}(\mathbf{Q})$, the latter being understood to include the nuclearrepulsion energy. Thus the requirement for a stationary point on the $J$-dependent effective potential-energy hypersurface becomes

$\nabla E_{\mathrm{e}}(\mathbf{Q})+\nabla E_{\mathrm{r}}(\mathbf{Q}, \mathbf{J})=0$

The rotational-energy gradients may, in turn, be expressed in terms of inertial derivatives; i.e. the derivatives of the components of the moment-of-inertia tensor with respect to the nuclear coordinates taken as internal coordinates. One representation of these coordinates is that given by Pulay and Sawodny [10]. Like the nuclear-repulsion energy gradient, the energy gradient is recalculated at each step of the geometry optimization.

In an analogous way the Hessian matrix, $\mathbf{H}$, may be augmented by a $\mathbf{J}$ dependent contribution from $E_{\mathrm{r}}$. Stationary points may be located which correspond to saddle points on the effective potential-energy hypersurface. Thus it is possible to explore the onset of rotationally induced symmetry-breaking instabilities.

A centrifugal stabilization energy, $\Delta E$, is defined, as before [6-9], as the difference between the energy of an assumed rigid molecule $\left(\mathbf{Q}=\mathbf{Q}_{0}\right.$, the usual equilibrium structure) with angular momentum $\mathbf{J}$ and that of the non-rigid molecule with the same $\mathbf{J}$

$\Delta E=\left[E_{\mathrm{e}}\left(\mathbf{Q}_{0}\right)+E_{\mathrm{r}}\left(\mathbf{Q}_{0}, \mathbf{J}\right)\right]-\left[E_{\mathrm{e}}(\mathbf{Q})+E_{\mathrm{r}}(\mathbf{Q}, \mathbf{J})\right]$

The resulting information maybe re-expressed, if desired, in terms of spectroscopic constants, as described previously [6-9]. 


\section{SUMMARY}

A general method is outlined for the augmentation of potential-energy hypersurfaces by classical rotational energies. Analytic gradient procedures may then be used to locate stationary points corresponding to $J$-dependent quasiequilibrium structures. The concomitant centrifugal stabilization energies may then be used to obtain centrifugal distortion spectroscopic constants.

\section{ACKNOWLEDGEMENT}

The author wishes to thank Professor Pulay of the University of Arkansas for several very helpful suggestions.

\section{REFERENCES}

1 J. Bordé, C. Bordé, C. Salomon, A. Van Lerberge, M, Ouhayoun and C. Cantrell, Phys. Rev. Lett., 45 (1980) 14.

2 J. Bordé and C.J, Bordé, Chem. Phys., 71 (1982) 417: 84 (1984) 159.

3 B. Bobin, C. Bordé, J. Bordé and C. Breant, J. Mol. Spectrosc., 121 (1987) 91.

4 D.J. Patterson, F. Herlement, M. Azizi and J. Lemaire, J. Mol. Spectrosc., 108 (1984) 31.

5 W.G. Harter and C.W. Patterson, J. Chem. Phys. 80 (1984) 4241.

6 L.L. Lohr and J.-M.J. Popa, J. Chem. Phys, 84 (1986) 4196.

7 L.L. Lohr and A.J. Helman, J. Comput. Chem, 8 (1987) 307.

8 L.L. Lohr, Int. J. Quantum Chem: Quantum Chem. Symp., 21 (1987) 407.

9 A. Taleb-Bendiab and L.L. Lohr, J. Mol. Spectrosc., 132 (1988) 413.

10 P. Pulay and W. Sawodny, J. Mol. Spectrosc., 26 (1968) 150. 